\title{
Gas Accretion and Galactic Chemical Evolution: Theory and Observations
}

\author{
Kristian Finlator
}

This chapter reviews how galactic inflows influence galaxy metallicity. The goal is to discuss predictions from theoretical models, but particular emphasis is placed on the insights that result from using models to interpret observations. Even as the classical "G-dwarf problem" endures in the latest round of observational confirmation, a rich and tantalizing new phenomenology of relationships between $M_{*}, Z, \mathrm{SFR}$, and gas fraction is emerging both in observations and in theoretical models. A consensus interpretation is emerging in which star-forming galaxies do most of their growing in a quiescent way that balances gas inflows and gas processing, and metal dilution with enrichment. Models that explicitly invoke this idea via equilibrium conditions can be used to infer inflow rates from observations, while models that do not assume equilibrium growth tend to recover it self-consistently. Mergers are an overall subdominant mechanism for delivering fresh gas to galaxies, but they trigger radial flows of previously-accreted gas that flatten radial gas-phase metallicity gradients and temporarily suppress central metallicities. Radial gradients are generically expected to be steep at early times and then flattened by mergers and enriched inflows of recycled gas at late times. However, further theoretical work is required in order to understand how to interpret observations. Likewise, more observational work is needed in order to understand how metallicity gradients evolve to high redshifts.

\section{Introduction}

Theoretically, inflows happen. The classical and perhaps most famous motivation for this view is the failure of the "closed-box" model (Schmidt, 1963; Tinslev, 1980) to account for the observed paucity of low-metallicity G and K stars (Pagel \& Patchett, 1975; Casuso \& Beckman, 2004), a discrepancy that persists even with the most

Kristian Finlator

New Mexico State University, Las Cruces, NM, USA

e-mail: finlatorenmsu.edu 
recent measurements of stars throughout the Milky Way disk (Schlesinger et al., 2012). The closed-box model has other problems such as its inability to account for the slow decline in galaxy gas fractions (Tacconi et al., 2013) and the cosmic abundance of neutral hydrogen (Wolfe et al., 2005). Likewise, it cannot account for the weak observed evolution in galaxy metallicities during the interval $z=2 \rightarrow 0$ (Erb et al., 2006), an epoch during which most of the present-day stellar mass formed.

More importantly, however, galaxy growth without inflows is theoretically incompatible with the current $\Lambda \mathrm{CDM}$ paradigm. In this picture, galaxies are viewed as condensations of cold baryons within dark matter halos. The dark matter halos themselves grow via a sequence of mergers that is decoupled from baryon physics and straightforward to compute using either analytic (White \& Rees, 1978; White \& Frenk, 1991) or numerical methods (Springel et al., 2005). As the halos grow, they accrete gas directly from the intergalactic medium (IGM). The vast majority of this gas accretes in a smooth fashion; that is, it does not arrive having previously condensed into an interstellar medium (ISM; Nelson et al. 2013). Halos that are more massive than the cosmic Jeans mass are expected to acquire a mass of baryons that is of order $\frac{\Omega_{b}}{\Omega_{-}} \times M_{\mathrm{DM}}$, where $M_{\mathrm{DM}}$ is the halo's total mass Gnedin, 2000; Okamoto et al., 2008; Christensen et al., 2016). Roughly half of this material collapses from the halo onto the central galaxy (Christensen et al., 2016), driving further star formation.

The expected thermal history of collapsed gas prior to its arrival in the central galaxy remains a topic of active study. It was originally assumed that all gas is shock-heated to the virial temperature and then cools in a spherically-symmetric way (White \& Rees, 1978). This was challenged a decade ago by numerical calculations, which found that much of the gas accretes directly onto the central galaxy without ever being heated, particularly at masses below $10^{12} M_{\odot}$ Kereš et al., 2005; Dekel \& Birnboim, 2006). The most recent calculations that include significantly improved hydrodynamic solvers contradict those results, attributing the lack of shock-heating and the inefficient cooling of the hot gas in previous calculations to numerical problems (Nelson et al., 2013). The new calculations indicate that the majority of gas at all halo masses is heated to the virial temperature before accreting onto the halo. However, it does not accrete in a spherically-symmetric fashion as originally envisioned (White \& Rees, 1978). Instead, it tends to concentrate in coherent structures that connect to large-scale intergalactic medium (IGM) filaments. The upshot is that, one way or another, gas readily accretes efficiently enough in $\Lambda \mathrm{CDM}$ to form the observed galaxy populations, with most gas arriving in the form of smooth inflows.

Once the gas condenses to densities of $\sim 1$ atom per $\mathrm{cm}^{-3}$, gravitational instability triggers the formation of molecular clouds and eventually stars. Feedback energy from the young stars limits the efficiency of star formation and regulates the ISM's structure in a number of ways. For our purposes, the most important of these is the generation of galactic outflows, which are inevitably observed wherever there is vigorous star formation (Veilleux et al., 2005). Theoretical models consistently predict that the mass of material that is ejected is comparable to or greater than the mass of 
stars that form (Murray et al., 2005; Muratov et al., 2015; Christensen et al., 2016). This enriched material then becomes available for re-accretion after a few dynamical times (Oppenheimer et al., 2010; Henriques et al., 2013; Christensen et al., 2016).

Outflows thus give rise to two conceptually distinct gas accretion channels, "Primordial Gas" and "Recycled Gas". Primordial gas dominates inflows at early times and low masses (Oppenheimer et al., 2010; Ma et al., 2016), and it dilutes galaxies' gas-phase metallicities. Recycled gas becomes increasingly important at late times and high masses. It is pre-enriched, and therefore less effective at dilution.

To summarize, in the era of $\Lambda \mathrm{CDM}$, galaxy growth driven by ongoing inflows is unavoidable. The central conceit of this chapter is that measurements of galaxy metallicities may be used to test models of those inflows. To motivate our discussion of how they do so, we list the observational probes that have been deployed:

- Stellar metallicity distributions;

- The slope, normalization, and evolution of the mass-metallicity relation (MZR);

- Third-parameter dependencies of metallicity on SFR, gas fraction, redshift, and environment; and

- Radial metallicity gradients (chiefly of the gas).

Stellar metallicity distributions have historically been an important indicator that inflows occur, but they are only available for the Milky Way and a handful of its satellite galaxies Kirby et al., 2011). For this reason, we will not discuss them further. Rather, we will focus on extragalactic diagnostics where larger samples are available. We also note that, throughout this discussion, we will focus on the oxygen metallicity as it is the most widely-observed tracer of the overall gas-phase metallicity. In Section 2, we review the physical processes through which inflows modulate galaxy metallicities. In Section 3, we discuss the extent to which galaxy growth tracks the host halo growth. In Section 4 , we introduce the Equilibrium Model, which is the simplest way for relating observables to inflows. In Section 5 we discuss departures from equilibrium growth. Finally, in Section 6 we summarize.

\section{Physical Processes}

Gas inflows impact galaxy metallicities on a wide range of timescales and spatial scales, and through a variety of processes that we associate either with Mergers, Galactic Fountains, or Environment. In this section, we summarize observational and theoretical progress in understanding these processes.

\subsection{Mergers}

Both observations and theory indicate that mergers are not the dominant way in which fresh gas is delivered to galaxies (Papovich et al., 2011; Behroozi et al., 
2013). However, on timescales comparable to a dynamical time, mergers can cause relatively unenriched gas that has previously settled in a galaxy's outskirts to plummet into the central 100-1000 parsecs (pc), simultaneously suppressing the central gas metallicity and boosting the central star formation rate.

This effect arises naturally in hydrodynamic simulations of galaxy mergers. Torrey et al. (2012) found that interactions suppress the nuclear metallicity of gas-poor galaxies (gas fractions of $\leq 20 \%$ ) by an amount that fluctuates depending on which merger stage the galaxies are observed in, but is typically $\approx 0.07$ dex. Gas-rich galaxies, by contrast, can experience nuclear metallicity boosts during interactions although this effect is not expected in typical SDSS galaxies owing to their generally low gas fractions. A similar result was presented by Rupke et al. (2010a), who found that the suppressed metallicities were associated with the period between the first and second pericenter passages of a merger event.

Does this mean that metallicities can be used to detect mergers? In fact, the statistical signature of nuclear inflows has been identified observationally in galaxy pairs from the Sloan Digital Sky Survey (SDSS). In particular,Scudder et al. (2012, 2013) showed that star-forming galaxies have suppressed central metallicity (by $0.02-0.3$ dex) and enhanced central SFR (by 60\%) if they are separated from a paired galaxy by a projected separation of $\leq 80 \mathrm{kpc} h^{-1}$ (see also Rupke et al. 2010b). It is also possible that galaxy mass and merger mass ratio modulate this effect: Splitting the merging SDSS galaxies by stellar mass, Michel-Dansac et al. (2008) have argued that mergers systematically boost the metallicity of low-mass galaxies while suppressing the metallicity of massive ones. The different behavior reflects a competition between dilution and enrichment, and is qualitatively expected based on the results presented by Rupke et al. (2010a) if low-mass galaxies are relatively gas-rich while massive ones are gas-poor.

While the ability of mergers to drive nuclear flows has been identified both in simulations and in observations, these flows have yet to be invoked as a rigorous constraint on galaxy evolution models. As a first step in this direction, Grønnow et al. (2015) asked whether a small but robust population of galaxies whose metallicities were lower than expected for their combination of stellar mass and star formation rate (Mannucci et al., 2010) could be interpreted as ongoing mergers or merger remnants. They developed a simple analytical model invoking the known halo merger rate along with the assumption that mergers boost SFR and suppress metallicity for a set time and showed that this model's parameters can be tuned to yield excellent agreement with the observed outlier population. They found that post-merger galaxies must exhibit diluted nuclear metallicities for $1.568_{-0.027}^{+0.020} \mathrm{Gyr}$, and the average dilution for mergers with mass ratios of 1:1-1:5 was 0.114 dex. Encouragingly, these inferences are within a factor of two of expectations from numerical simulations and observations.

A caveat to analyses of SDSS metallicities is that the SDSS spectroscopic fibers subtend a diameter of 3 arcsec, hence they capture only the central $5 \mathrm{kpc}$ of galaxies at the typical redshift of SDSS galaxies $(z=0.1)$. While this makes them ideal for studying nuclear metallicities, it leaves unanswered the question of how mergers effect the metallicity at larger radii. Rupke et al. (2010a) showed that the same 
interaction-triggered flows that dilute central metallicities also flatten overall gas metallicity gradients. This effect has likewise been observed in ongoing mergers (Kewley et al., 2010). Unfortunately, it is difficult to use the physics of ongoing mergers to test galaxy formation models because results are so sensitive to details of the merger such as gas fractions, mass ratio, and orbital parameters (Torrey et al., 2012).

Fortunately, the tendency for mergers to flatten metallicity gradients and to build up central bulges does leave an observable signature: Galaxies that are more massive or have larger (classical) bulges should have flatter gas metallicity gradients. Fu et al. (2013) have shown that this effect is strong observationally, and can readily be reproduced by their semi-analytical model (their Figure 12). However, they did not explore which of the assumptions underlying their merger model are required to yield the good agreement. Moreover, they address only local observations, and suggest that extending the work to higher redshifts would be a useful next step. We will return to this point in Section 4.2

\subsection{Outflows and Galactic Fountains}

The way in which galactic inflows modulate metallicities and metallicity gradients depends critically on galactic fountains. Observations indicate that vigorous star formation is inevitably associated with outflows that eject gas from galaxies at a rate that is comparable to the star formation rate (Veilleux et al., 2005). Observations and models both suggest that they carry away most of the metals that are generated in core-collapse supernovae (Martin et al., 2002; Fu et al., 2013). The gas is expected to re-accrete on a dynamical timescale, particularly in massive galaxies $\left(\log \left(M_{*} / M_{\odot}\right)>10.5\right)$ and at relatively late times $(z<1$; Oppenheimer et al. 2010; Henriques et al. 2013). The gas that a central galaxy re-accretes contains contributions both from its progenitor and from satellite systems, while satellite systems probably do not accrete much gas. Importantly, while the ejected gas has predominantly low angular momentum, it picks up angular momentum from the halo and re-accretes at generally larger radii than where it was ejected (Brook et al., 2012; Christensen et al., 2016). This has a number of observational consequences.

\subsubsection{Galaxy-averaged quantities}

First, re-accreted metals boost the central galaxy's metallicity with respect to models with unenriched inflows (or, viewed from a different perspective, they suppress dilution). If the metallicity of inflowing gas varies with mass, then recycled gas must

affect the slope of the mass-metallicity relation. In particular, if low-mass galaxies accrete predominantly pristine material while inflows into massive galaxies are preenriched (Ma et al., 2016; Brook et al., 2014), then this differential pre-enrichment steepens the slope of the mass-metallicity relation. 
If pre-enrichment levels vary with redshift, then they furthermore contribute to the evolution of the normalization of the mass-metallicity relation (Davé et al., 2011). For example, if inflows are pristine at early times and enriched to roughly the same level as the ISM at $z=0$, then they are more effective at diluting gas reservoirs at high redshift, driving stronger evolution in the normalization of the mass-metallicity relation. The normalization of the MZR is observed to increase (Maiolino et al., 2008; Faist et al., 2016), which could be explained in this way. The observational challenge is to disentangle this factor from other influences that could also evolve with redshift (or mass) such as the initial mass function (van Dokkum, 2008; Davé, 2008).

It is instructive to compare this interpretation of how the MZR's normalization evolves upwards to that presented in Ma et al. (2016). In the latter work, highresolution simulations were used to show that the relationship between gas metallicity and stellar mass fraction $f_{*}$ (that is, the stellar mass divided by the baryonic mass) is nearly that of a closed-box throughout $z=3 \rightarrow 0$ when averaged over the entire halo rather than just the central galaxy. In this view, growth in the MZR's normalization tracks growth in $f_{*}$. While this is a suggestive insight, it does not directly address growth in galaxy metallicities, which are far more readily observable. It is not hard to imagine, for example, that gas flows might hide metals within the halo at high redshifts but then shift progressively more of them into observability at late times. In other words, galaxy metallicities may evolve to become an increasingly unbiased probe of the halo metallicity, which in turn tracks closed-box expectations. A more detailed study of the evolving relationship between halo-averaged and ISMaveraged metallicity is probably indicated. For the present, however, it is clear that pre-enriched inflows have the potential to drive MZR evolution.

\subsubsection{Radial Metallicity Gradients}

If inflows deposit gas that has relatively uniform metallicity over a range of radii as expected theoretically (Brook et al., 2012; Christensen et al., 2016), then they flatten radial metallicity gradients because the metallicity at any point is driven by the inflowing gas, washing out other influences such as radial gradients in star formation efficiency or wind characteristics, or radial flows that escort low-metallicity in from the galaxy's outskirts. This raises the possibility of using observed metallicity gradients to constrain inflows.

At low redshift, it has long been known observationally that star-forming galaxies have slowly-declining metallicity gradients (Zaritsky et al., 1994), and recent analyses have confirmed these results (Fu et al., 2013; Carton et al., 2015; Ho et al., 2015). Fu et al. (2013) used a semi-analytical model of galaxy formation to interpret observations. They showed that inflows can readily dominate metallicity gradients (their Figure 6), while the effect of radial flows is probably relatively weak. They further found that it was necessary to assume that $80 \%$ of all newlyformed metals are launched into the halo in order to match observed metallicity gradients, qualitatively consistent with inferences from X-ray observations of local 
outflows (Martin et al., 2002). In their model, the tendency for ejected metals to reaccrete over a range of radii makes the baryon cycle into an efficient method for redistributing metals.

Carton et al. (2015) interpreted locally-observed metallicity gradients using a simpler model that assumes a local equilibrium between inflows, star formation, and outflows (Section 4.2). In their model, radial flows are ignored, and inflows are assumed to be uniform across the disk. Hence the observed metallicity gradient is driven by the radial dependence of the mass-loading factor (that is, the ratio of the outflow to star formation rates), with weaker outflows yielding higher metallicity in the center and stronger outflows suppressing it toward the disk edge. This model requires inflows in order to balance ongoing enrichment (Lilly et al., 2013), but that does not mean that the data require strong inflows. In fact, it is not even possible to use the Carton et al. (2015) model to measure inflow rates or metallicities owing to degeneracies. For example, a high observed metallicity could reflect a high metal yield and unenriched inflows, or a low metal yield and highly-enriched inflows.

The model explored by Ho et al. (2015) leads to a different interpretation, tying locally-observed metallicity gradients to gradients in gas fraction. These authors relax the assumption that all regions of a galaxy are in local enrichment equilibrium, but assume that the mass-loading factor and the ratio of inflow to star formation rates $\dot{M}_{g, \text { in }} /$ SFR are all constant. In this case, metallicity decreases with radius because more diffuse regions have higher gas fraction, implying that they are chemically less mature. They also find that their models fit observations if they assume that both inflows and outflows are weak, indicating nearly closed-box chemical evolution for systems with low gas fraction.

The Ho et al. (2015) and Carton et al. (2015) studies both leverage high-quality measurements of radial trends in metallicity and gas fraction, but their modeling efforts lead to different conclusions regarding the flow of gas into and out of the galaxy because they invoke different assumptions. Which model is more correct? More theoretical insight into how observables connect to the underlying physics would certainly help. At the same time, it is to be hoped that future studies that leverage measurements on halo metallicities or inflow rates (from, for example, absorptionline campaigns) will eventually break the underlying degeneracies.

Fu et al. (2013) indicated that the evolution of metallicity gradients to high redshift is a complementary constraint on star formation and gas flows. This echoes Pilkington et al. (2012), who analyzed how the radial and vertical gradients evolved in 25 cosmological simulations of Milky Way analogs from several groups as well as two independent analytical models. They found that, although all models roughly reproduce the Milky Way's current radial gradient, some predicted dramatically steeper gradients at earlier times while others did not. They concluded that steep gradients in metallicity reflect steep gradients in star formation efficiency and noted that strong feedback can wash out metallicity gradients. However, their discussion did not consider the possible role of inflows in balancing enrichment.

Observationally, the high-redshift story is far from clear. An early integral field study of three star-forming galaxies at $z \sim 3$ uncovered inverted metallicity gradients (i.e., the gas-phase metallicity is lower in the center; Cresci et al. 2010). The 
authors interpreted their findings as evidence that inflows deliver pristine gas preferentially to the center of high-redshift galaxies, although in fact this is not expected to produce inverted gradients generically (Pilkington et al., 2012).

Over the following years, detailed analyses of a few strongly-lensed high-redshift systems yielded steeply declining metallicity gradients (Yuan et al., 2011; Jones et al., 2013) as predicted in Pilkington et al. (2012). These results seemed to indicate that strong inflows do not flatten or invert metallicity gradients at high redshifts.

Most recently, however, a study of a large sample of unlensed systems indicated that metallicity gradients are flatter than in the local Universe or even absent at higher redshifts (Wuyts et al., 2016). As noted in Wuyts et al. (2016), it is not obvious why lensed systems should show strong gradients while unlensed ones do not, particularly given that the samples overlap in stellar mass. Wuyts et al. (2016) consider a number of effects such as beam-smearing, AGN, or shocks that could artificially suppress the intrinsic metallicity gradient in unlensed systems, but conclude that they would have detected strong gradients if they were there. Hence while further work is needed to control biases, current observations support the idea that strong inflows flatten high-redshift metallicity gradients, as qualitatively suggested by some (but not all) models (Brook et al., 2012; Fu et al., 2013).

\subsubsection{Future Work}

At a technical level, the pioneering study of re-accretion presented in Oppenheimer et al. (2010) deserves to be re-visited in the context of more recent numerical models for two reasons. The less important of these is that galactic outflow models have grown more realistic owing to high-resolution simulations (Muratov et al., 2015) as well as to increasingly detailed comparison with measurements (Mitra et al., 2015). The more important reason is that simulations now incorporate significantly improved hydrodynamic solvers and dynamic range, which are critical for resolving the complicated interaction between outflows and the circumgalactic medium (Nelson et al., 2015). Relatedly, improved cross-fertilization of insights regarding outflows and enriched inflows between hydrodynamic simulations and semi-analytical models would be helpful both for distilling insight from the numerical models and for exploring its implications within a more flexible framework.

With an improved understanding of the baryon and metal cycles, a straightforward next step would be to review the hypothesis presented in Davé et al. (2011) that pre-enriched inflows drive the MZR's shape and evolution. A detailed budgeting of how galaxies distribute their baryons and metals within the ISM and halos would be necessary for this step, and would inform the next generation of measurements of the CGM, which has become a very active field over the past few years.

In the specific case of merger-induced inflows, it is not to soon to ask whether simulations can accommodate the rich set of observations of how interactions trigger nuclear flows driving star formation, gas dilution, as well as AGN activity (cf. the "Galaxy Pairs in the Sloan Digital Sky Survey" paper series by S. Ellison and 
collaborators); this would test the hypothesis that mergers can be identified based on their suppressed nuclear metallicities (Grønnow et al., 2015).

Finally, the effective use of metallicity gradients for constraining gas inflows in a cosmological context will require further development both in models and in observations. An improved understanding of what drives the metallicity gradients that are predicted by numerical simulations, a converged picture for the observed evolution of metallicity gradients to higher redshift, and the continued development of techniques for using observations to test models (for example, accounting for biases associated with sample selection and and strong-line abundance indicators) will yield further insight into the baryon cycle.

\subsection{Environment}

At fixed stellar mass, galaxies that live in richer environments are observed to have slightly higher metallicities (Mouhcine et al., 2007; Cooper et al., 2008), although the effect is weak and not always observed (Hughes et al., 2013). A qualitatively similar relationship occurs in cosmological simulations (Davé et al., 2011), suggesting that it may in fact be real. If so, it could reflect systematic variation in the basic properties of the baryon cycle. For example, it is easy to imagine that weaker outflows, more efficient re-accretion of previously-ejected gas, or enriched inflows could boost the metallicities in overdense regions. Indeed, analytical models have been used to argue that enriched inflows are a viable explanation for the offset (Peng \& Maiolino, 2014).

In reality, it may be that none of these is the correct explanation. A detailed study of galaxies in the ILLUSTRIS simulations (Genel, 2016) recently traced the metallicity-environment correlation to two causes. First, at a given stellar mass, satellite galaxies (which dominate richer environments) tend to form earlier than centrals, draining their gas reservoirs and boosting their metallicities. Second, satellites' disks tend to be truncated and more centrally-concentrated such that observations are weighted toward their metal-rich cores. Combining these effects essentially accounts for the the entire simulated offset.

Genel (2016) notes that the CGM around satellite galaxies is more enriched than around centrals of the same stellar mass, and in fact the offset is comparable to offset in the galaxies' metallicities. However, he argues that this does not dominate the dependence of metallicity on environment because centrals and satellites with similar stellar mass and star formation history have nearly the same metallicitydespite the apparently higher metallicity of the gas around satellites.

Future work disentangling the metallicity of inflowing and outflowing gas may be needed in order to understand how a more enriched CGM does not drive higher galaxy metallicities. For the present, however, it seems that the tendency for metallicity to increase in rich environments does not relate to inflows. 


\section{Galaxy Growth and Halo Growth}

The previous section drew attention to our imperfect understanding of how galaxies and halos grow together. A specific problem related to this question is the "dwarf galaxy conundrum", or the suite of observational clues that galaxies with stellar masses below $10^{10} M_{\odot}$ do not grow at the same specific rate as their host halos (for example, Weinmann et al. 2012). This behavior is very difficult for conventional galaxy evolution models to accommodate (White et al., 2015; Somerville \& Davé, 2015): generically, the strong outflows that seem required in order to bring the predicted stellar mass function at $z=0$ into agreement with observations lead to metallicities and gas fractions that are under-predicted at low redshifts. Conversely, adopting weaker outflows at low masses in order to match MZR observations yields too many stars in low-mass halos at $z=0$.

The problem clearly points to the need for a qualitatively new physical mechanism that retards gas processing in low-mass systems. This raises the question of whether that process operates in the IGM/CGM, or in the ISM. In a creative analytic model, Bouché et al. (2010) argued for a CGM-based solution, showing that forbidding halos less massive than $10^{11} M_{\odot}$ from cooling their gas onto galaxies improves agreement with measurements of star formation and stellar mass growth. They unfortunately did not address metallicities. Lilly et al. (2013) also addressed this problem. They found that, under the assumption that enrichment and dilution balance exactly (Section 4), the fraction of galaxy gas that is converted to stars can be inferred directly from the MZR, and the resulting scaling matches the requirement that is implied by the stellar mass function. In this argument, the dwarf galaxy conundrum can be resolved by processes that occur within the ISM, although inflows are required.

In order to assault the problem using a more comprehensive suite of observables, White et al. (2015) implement several galaxy-slowing mechanisms (both galaxy-based and CGM-based) into a semi-analytical model. They show that two changes to the fiducial model can improve agreement: in their "preferential reheating" model, low-mass galaxies eject systematically more gas in outflows per unit of stellar mass formed at higher redshift. In their "parking lot" model, ejected gas is not permitted to re-accrete for a delay time that depends on halo mass (see also Henriques et al. 2013). By manipulating the parameters governing these processes, they found improved agreement with measurements of cold gas fractions, specific star formation rates, and ISM metallicities

In the context of numerical simulations, Ma et al. (2016) have shown that accounting more realistically for the physical processes that occur within the interstellar medium (ISM) tends naturally to decouple the growth of low-mass galaxies from their host halo, leading to improved agreement with observations of the MZR evolution (see also Hopkins et al. 2014). Their findings are qualitatively consistent with the inferences that White et al. (2015) draw from semi-analytical models, but it is difficult to draw robust conclusions owing to the small number of halos that have been simulated at high resolution. For example, is the bottleneck that retards gas processing located in the ISM, in the CGM, or both? Ongoing efforts to distill 
their results into scalings that can be implemented into cosmological simulations and semi-analytical models will generate further insight (Davé et al., 2016).

\section{The Equilibrium Model}

Over the last decade, an increasing number of theoretical studies have arrived at the conclusion that reasonably massive star-forming galaxies $\left(M_{*}>10^{10} M_{\odot}\right)$ grow in a quasi-smooth fashion whereby gas is accreted from the CGM and processed at roughly the same rate. This is a generalization of the idea that star formation self-regulates to match the gas accretion rate (Tinsley \& Larson, 1978; Köppen \& Edmunds, 1999) in that it replaces "star formation" with "gas processing", where by the latter we mean that gas is processed either into stars or outflows. In this section, we introduce the concepts governing the equilibrium model and discuss inquiries into when and where it applies.

\subsection{A Single Zone}

We begin by considering a galaxy to be a single zone and define the growth rate of stellar mass in long-lived stars $\dot{M}_{*}$, the rate of change of the gas mass $\dot{M}_{g}$, the gas and star accretion rates $\dot{M}_{g, \text { in }}, \dot{M}_{* \text {,in }}$, and the gas outflow rate $\dot{M}_{g, \text { out }}$. Generically, these are related by

$$
\dot{M}_{*}+\dot{M}_{g}=\dot{M}_{g, \text { in }}-\dot{M}_{g, \text { out }}+\dot{M}_{*, \text { in }}
$$

Equation 1 is a tolerably comprehensive statement of the conservation of baryonic mass within the context of galaxy evolution. Note that star formation does not appear in this equation because it does not change the galaxy's baryonic mass.

To derive the Equilibrium Model, we begin by assuming that accretion of already-formed stars in actively star-forming galaxies is negligible; that is, $\dot{M}_{*, \text { in }}=0$. This implies that stellar mass growth owes predominantly to star formation, or $\dot{M}_{*}=$ SFR, which is supported observationally (Papovich et al., 2011; Behroozi et al., 2013). Next, we assume that the outflow rate equals the star formation rate of long-lived stars multiplied by a slowly-varying "mass-loading factor" $\eta$; that is, $\dot{M}_{g, \text { out }}=\eta \dot{M}_{*}$. Finally, we assume that the galaxy balances accretion exactly with gas processing so that $\dot{M}_{g}=0$. This condition cannot, of course, be exactly correct, as it would imply that galaxies have no gas. However, high-resolutions simulations suggest that, indeed, the mass of material that is processed and ejected by disks is comparable to the mass that is accreted (Christensen et al., 2016), justifying it as a first-order approximation. What is left is (Finlator et al., 2008):

$$
\dot{M}_{*}(1+\eta)=\dot{M}_{g, \text { in }}
$$


For clarity, we note that many authors define a total star formation rate SFR, compute the "return fraction", $R$, or the fraction of mass that collapses into massive stars and is then returned to the ISM on a short timescale (Tinsley, 1980), and then write down the growth of stellar mass in long-lived stars as $\dot{M}_{*}=\operatorname{SFR}(1-R)$. In this case, we define $\eta$ as the the ratio of the outflow rate to the total star formation rate, and Equation 2 is unchanged.

Equation 2 is a statement of gas processing equilibrium. The intuition is that, if the gas accretion rate temporarily exceeds the gas processing rate, then the gas density increases, boosting gas processing until the excess has been worked through. Conversely, if accretion temporarily sputters, then the gas density declines, choking off gas processing until new inflows are available. Equation 2 assumes that this balancing occurs on a timescale that is very short. In doing so, it departs conceptually from the traditional view that the star formation rate is fundamentally determined by the gas density. In an equilibrium scenario, the ISM adjusts itself so as to satisfy Equation 2, the gas density merely reflects the details of how it achieves this.

The factor of $(1+\eta)$ is what distinguishes Equation 2 from classical analytical models (Tinsley \& Larson, 1978; Köppen \& Edmunds, 1999). To see how important it is, consider the influences that determine a galaxy's gas-phase metallicity. At any given time, inflows from the CGM dilute the galaxy's metallicity. However, the inflows trigger continuing star formation, which simultaneously enriches the gas. The competition between dilution and enrichment gives rise to an Equilibrium Metallicity $Z_{\mathrm{eq}}$ (Finlator et al., 2008; Davé et al., 2012):

$$
Z_{\text {eq }} \equiv Z_{0}+\frac{y}{(1+\eta)}
$$

Here, $y$ is the metal yield, or the mass of metals ejected by core collapse supernovae per mass of long-lived stars formed; and $Z_{0}$ is the metallicity of inflowing gas, which for convenience we may re-cast as $Z_{0} \equiv \alpha_{Z} * Z$, where $\alpha_{Z}$ is the ratio of the metallicity of inflowing gas to the ISM metallicity and $Z$ is the ISM metallicity. If $\eta$ is redefined such that $\eta \equiv \mathrm{SFR} / \dot{M}_{g \text {,out }}$ where SFR is the total star formation rate, then we replace $\eta \rightarrow \eta /(1-R)$ in Equation 3 . Note that we assume that outflows have the same metallicity as the ISM; this is supported by high-resolution simulations (for example, Figure 11 of Ma et al. (2016).

Equation 3 is a statement of enrichment equilibrium. The intuition is that enrichment and dilution balance each other once the galaxy's metallicity reaches $Z_{\text {eq. }}$. If $Z$ somehow jumps above $Z_{\text {eq }}$, then inflows dilute it. If $Z$ dips, then continued star formation boosts it back to $Z_{\mathrm{eq}}$. If the SFR responds instantaneously to fluctuations in the inflow rate, then departures from $Z_{\mathrm{eq}}$ are erased on a dilution time $M_{g} / \dot{M}_{g, \text { in }}$, or the timescale for inflows to replenish the ISM completely.

Equations 2 3 involve two parameters, $\eta$ and $Z_{0}$. For galaxies that are massive enough to be in equilibrium, the slope and redshift-dependence of the massmetallicity relation may be expressed as evolution in these parameters.

In order to illustrate how powerful Equation 3 is, we use it to interpret the $z=0$ mass-metallicity relation (where by "metallicity" we mean the oxygen mass fraction $Z_{\text {ox }}$ ). In each panel of Figure 1, the black dashed line indicates $Z$ under 


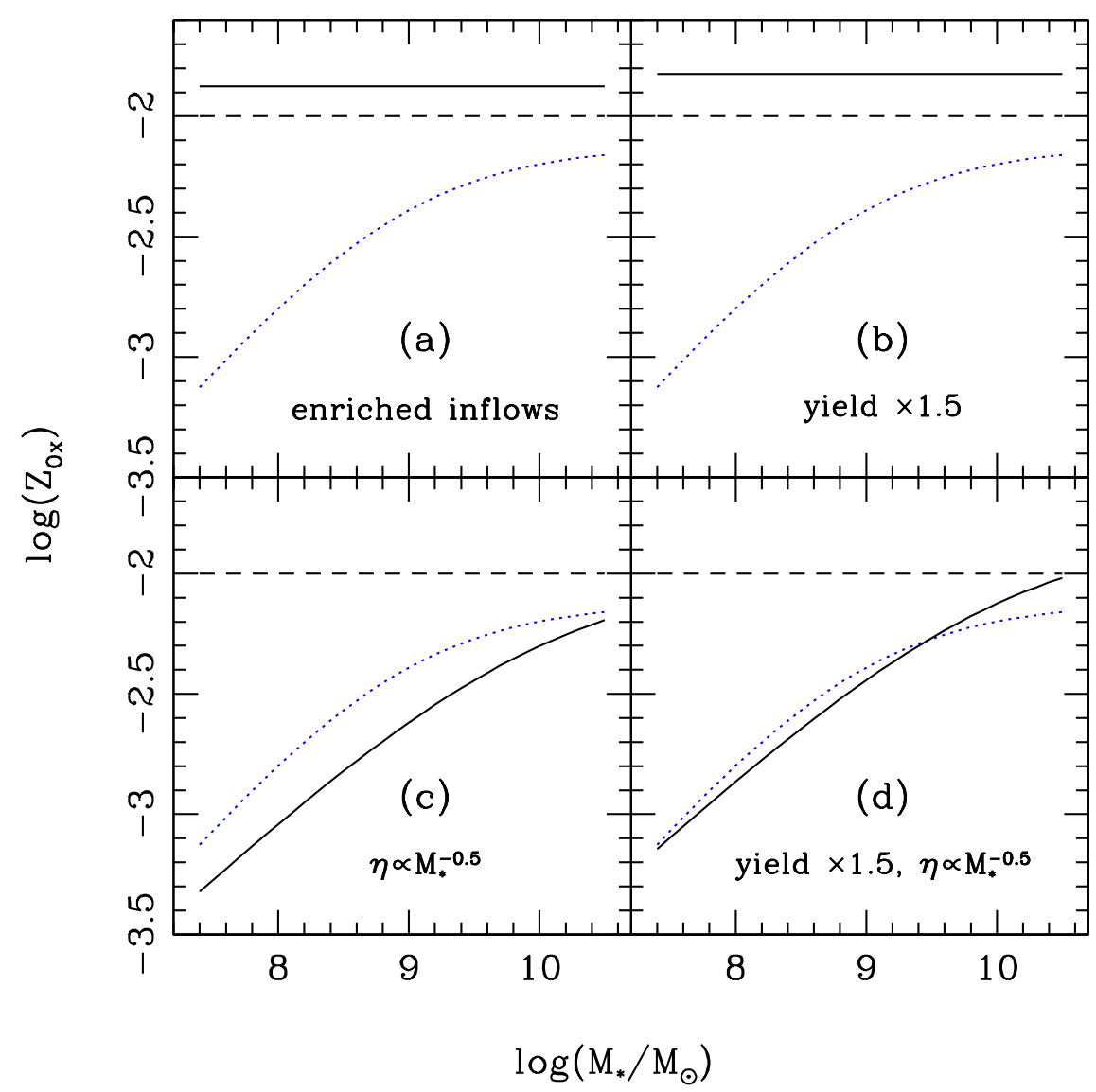

Fig. 1 Illustration of the parameters that control metallicity in Equation 3 In each panel, the black dashed line indicates the same "base model" while the blue dashed curve is the observed trend (Andrews \& Martini, 2013). The bottom-right panel shows that Equation 3 can be tuned to achieve good agreement with observations.

the assumption of pristine inflows $\left(Z_{0}=0\right)$, an oxygen yield of $y=0.01$, and no outflows $(\eta=0)$. Meanwhile, the blue dotted curve is the observed trend at $z \sim 0.1$ (Andrews \& Martini, 2013), where we convert from units of $12+\log (\mathrm{O} / \mathrm{H})$ to oxygen mass fraction assuming a hydrogen mass fraction of 0.76 .

With no outflows $(\eta=0)$, metallicity has no dependence on mass as shown by the dashed black segment in each panel of Figure 1, Increasing the inflow metallicity $Z_{0}$ from 0 to 0.00333 boosts $Z$ at all masses (panel a). Likewise, increasing the yield $y$ from 0.01 to 0.015 increases $Z$ at all masses (panel b). Assuming that the outflow rate $\eta$ varies with stellar mass as $\left(10^{10} M_{\odot} / M_{*}\right)^{0.5}$ immediately yields a strong dependence of $Z$ on $M_{*}$ (panel c). Finally, restoring the assumption of pris- 
tine inflows and tuning both the yield and the outflows yields a plausible level of agreement with observations (panel d). We do not use a rigorous approach to tune our parameters because our goal is merely to illustrate its potential as a conceptual framework. However, the model has been generalized and tuned elsewhere, yielding promising insight into the nature of the baryon cycle (Mitra et al., 2015, 2016); we will discuss these efforts shortly.

This exercise shows that the MZR can easily be interpreted within the equilibrium scenario (Equations 2-3). However, the interpretation's value is limited by model's simplicity. For example, $\eta$ and $Z_{0}$ could vary with redshift, and $Z_{0}$ could additionally vary with mass. For improved realism, further insight from numerical models is required.

$\eta$ is now relatively well-studied theoretically, with a consensus that it decreases with increasing mass and exceeds 1 in halos whose circular velocity $v_{\text {circ }}$ lies below $60 \mathrm{~km} \mathrm{~s}^{-1}$ (Muratov et al., 2015; Christensen et al., 2016). In detail, there is an ongoing debate as to whether it follows expectations from momentum-driven or energy-driven outflows. Briefly, if the characteristic speed of galactic outflows is the halo circular velocity $v_{\text {circ }}$ and if star formation deposits a constant amount of energy in outflows per unit of stellar mass formed, then $\eta$ varies as $v_{\text {circ }}^{-2}$. Alternatively, if star formation deposits a constant amount of momentum in outflows per unit of stellar mass formed, then $\eta \propto v_{\text {circ }}^{-1}$ (Murray et al., 2005).

We show three recent estimates for $\eta$ in Figure 2, Muratov et al. (2015) use highresolution numerical simulations to find that $v_{\text {circ }}^{-1.0}$ for $v_{\text {circ }}>60 \mathrm{~km} \mathrm{~s}^{-1}$ and $v_{\text {circ }}^{-3.2}$ at lower masses. By contrast, Christensen et al. (2016) study a complementary suite of high-resolution simulations and derive $\eta \propto v_{\text {circ }}^{-2.2}$ at all masses. These simulations model star formation and feedback in different ways, hence it is telling that they both predict $\eta>1$ for $v_{\text {circ }}<60 \mathrm{~km} \mathrm{~s}^{-1}$ and $\eta>4$ for $v_{\text {circ }}=30 \mathrm{~km} \mathrm{~s}^{-1}$, which is the lowest halo mass that can efficiently accrete baryons from a photoheated IGM (Gnedin, 2000; Okamoto et al., 2008). The generally higher values of $\eta$ found by Muratov et al. (2015) probably reflect additional feedback sources such as radiation pressure that are not considered in the Christensen et al. (2016) model.

Mitra et al. (2015) take a different approach and use a Bayesian Monte Carlo Markov Chain approach to infer $\eta$ (among other things) directly from observations. In particular, they assume that galaxies obey Equations $2-3$ at all times and then infer the parameters that are required in order to match simultaneously the observed stellar mass - halo mass relation, the MZR, and the stellar mass - star formation rate relation. They find that $\eta \propto M_{h}^{-1.16} \propto v_{\text {circ }}^{-3.48}$, slightly steeper than the dependencies obtained from numerical simulations. When $\eta$ is expressed as a function of stellar mass, however, the differences shrink: Muratov et al. (2015) find $\eta \propto M_{*}^{-0.35}$ while Mitra et al. (2015) find $\eta \propto M_{*}^{-0.5}$ for $M_{*}<10^{10} M_{\odot}$ (note that this is the same scaling as in panel d of Figure 1).

Given that $\eta$ decreases with mass both in ab-initio calculations and in efforts to model observations, it assuredly drives the MZR's slope: As shown in Figure 1, a large $\eta$ removes more enriched gas, with the result that dilution by inflows is more efficient at suppressing the gas metallicity. This in turn leads to a positive dependence of $Z$ on mass. 


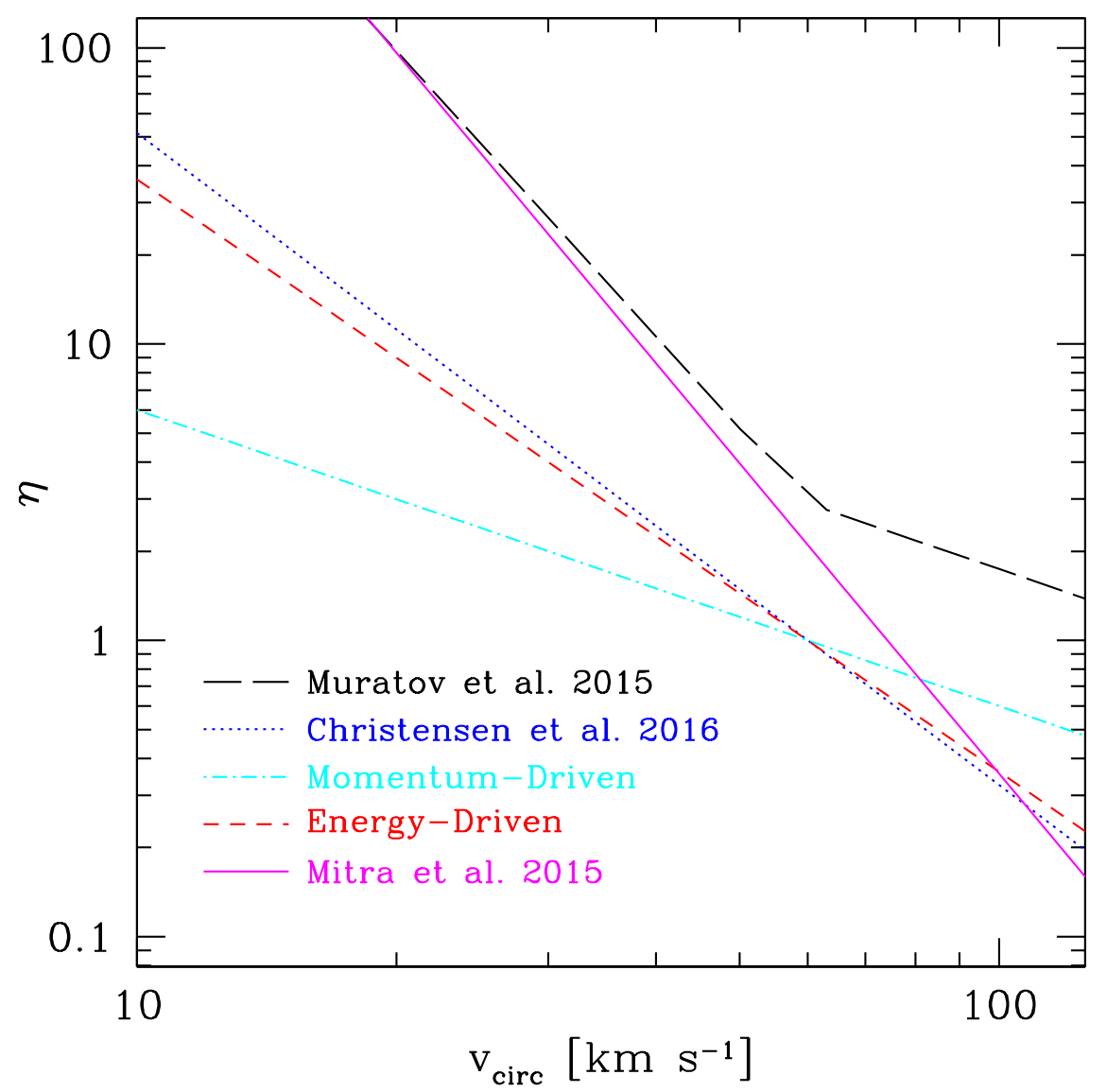

Fig. 2 Recent models for the ratio $\eta$ of the gas outflow rate to the star formation rate (a.k.a. the mass-loading factor). The models of Christensen et al. 2016 and Muratov et al. 2015 are highresolution ab-initio simulations. The Mitra et al. 2015 curve results from fitting an equilibrium model to observations. The momentum-driven and energy-driven scalings are arbitrarily normalized to $\eta=1$ at $60 \mathrm{~km} \mathrm{~s}^{-1}$.

The way in which enriched inflows (that is, $Z_{0}>0$ ) affect the MZR has received considerably less study than $\eta$ but is probably just as important. Davé et al. (2011) showed that, on average, $\alpha_{Z}$ increases with time, driving or at least contributing to the observed growth in the MZR's normalization. The analysis of metal flows in high-resolution simulations presented by Ma et al. (2016) qualitatively supports this view. Furthermore, it suggests that inflows into massive halos (those that grow to $10^{11} M_{\odot}$ by $z=0$ ) have roughly one third the ISM's metallicity at all times, or $\alpha_{Z} \sim \frac{1}{3}$. Meanwhile, the $\alpha_{Z}$ in low-mass halos $\left(10^{10} M_{\odot}\right.$ at $\left.z=0\right)$ falls from $\sim \frac{1}{3}$ at early times to a few percent at $z=0$. 
In short, both $\eta$ and $Z_{0}$ probably vary in a way that drives an MZR to increase with mass and time. The connection between this differential dilution and the MZR depends critically on the equilibrium model. This discussion then raises several points for future research.

Which galaxies are in equilibrium? As discussed in Section 3, observations suggest that low-mass halos depart from equilibrium expectations in the sense that they do not cool their gas onto galaxies at the same rate as they accrete it from the IGM. Is there a similar mass cutoff below which galaxies do not convert their stars into gas at the same rate as they accrete it from the CGM? Does it vary with redshift? If so, then one expects the MZR normalization to plummet above the redshift at which galaxies first achieved equilibrium. Indeed, observations have suggested that the MZR drops rapidly in normalization from $z=2-3$ (Maiolino et al., 2008) and then flattens (that is, the mass dependence weakens) to $z=5$ (Faist et al., 2016). This behavior could indicate that $z \geq 3$ is the "gas accumulation" epoch, when metal and dust production were swamped by inflows and $\eta$ and $\alpha_{Z}$ did not yet govern metallicities. A detailed analysis of what is expected from hydrodynamic simulations in this regard would be illuminating.

What is equilibrium? For completeness, we note that, while some works define gas processing equilibrium as the condition that a galaxy's gas mass be constant Finlator et al. (2008); Davé et al. (2011, 2012), it may be equally defensible to propose that a galaxy's gas fraction [defined as $\mu \equiv M_{g} / M_{*}$ or $M_{g} /\left(M_{g}+M_{*}\right)$ ] is constant (Feldmann, 2013). If true, then the equilibrium metallicity is slightly different from Equation 3 (i.e., set $\dot{\mu} \equiv 0$ in Equation 4 ). It would be interesting to ask which of these conditions more accurately describes galaxy growth in cosmological simulations or semi-analytical models.

Can we close the loop? Once the mass and redshift range has been identified where galaxies are expected to be in equilibrium, it would be interesting to ask whether Equations 2 really do govern the observed evolution within simulations and models. Conceptually, this would be accomplished in several (rather involved) steps:

- Identify which galaxies obey Equation 2 or an analogous statement of gas processing equilibrium such as $\dot{\mu}=0$;

- Measure $\eta\left(M_{*}, z\right), Z_{0}\left(M_{*}, z\right)$ and the MZR and its evolution;

- Ask whether Equation 3 or an analogous statement of enrichment equilibrium correctly describes the predicted MZR evolution.

A more complete conceptual understanding of what governs the MZR in hydrodynamic simulations or semi-analytical models would inform the interpretation of existing and upcoming observations. 


\subsection{Multi-zone models}

If the equilibrium model describes galaxies as a whole, then it is natural to ask whether it also describes individual regions within the galaxy. There is a lower limit of course; we would not apply the equilibrium model on spatial scales comparable to a molecular cloud (10-100 pc) where metals diffuse or are spread by individual supernovae. But what about a region of characteristic size $\sim 1 \mathrm{kpc}$ ? To be specific, we may ask two questions:

1. What is the smallest region within a galaxy whose gas reservoir obeys Equations 23. This is important because the relative roles of inflows and outflows are more difficult to assess (or even discuss) in systems that are not in equilibrium.

2. How does the balance between radial flows, outflows, and inflows vary within a star-forming galaxy?

Within the context of an analytical model, Fu et al. (2013) found that radial flows do not impact the overall metallicity gradient while inflows do. They did not study the extent to which individual annuli were in equilibrium, and the assumptions that were necessarily to make their model tractable were strong, motivating further insight from numerical models.

A year later, a different group took the bait and carried out a detailed study of high-resolution, one-dimensional simulations of star-forming disks (Forbes et al., 2014a). They found that, indeed, individual annuli do obey Equation 2 locally. In detail, the way in which cosmological inflows, star formation, galactic outflows, and radial gas flows (triggered by gravitational instability-driven torques that occur wherever gas densities are high) balance varies with mass and with redshift. Annuli at large radii have low densities and low star formation rates, hence they do not efficiently process newly-accreted gas or transport it to smaller radii. Moving inwards, gas densities increase to the point that star formation and radial transport of gas balance accretion. Near a galaxy's center, star formation and outflows are so efficient that they can only be balanced by radial transport of gas from larger radii; cosmological inflows are a subdominant gas source here. The slowly-declining cosmological infall rate causes this balance to evolve in such a way that the region where gas is gravitationally unstable migrates outwards with time. By $z=0$ it operates only in galaxies' outskirts, if at all.

The finding that high-redshift galaxies obey Equation 2 locally as well as globally certainly raises the hope that Equation 3 also applies, but Forbes et al. (2014a) unfortunately do not comment on the predicted metallicity gradients or their evolution. However, simple applications of this idea have been able to accommodate locally-observed radial trends fairly well (Carton et al., 2015).

It would be interesting to compare high-resolution one-dimensional simulations (Forbes et al.,2014a) with lower-resolution cosmological simulations (Pilkington et al., 2012) in order to assess (1) Whether cosmological simulations resolve gravitational instabilities and their impact; (2) Whether there are effects in three-dimensional simulations (such as bars) that modulate gas flows and that cannot be captured in 
one-dimensional simulations; and most broadly (3) Whether the cosmological simulations predict the same level of equilibrium as the one-dimensional simulations. Likewise, it would be useful to distill insights from the Forbes et al. (2014a) study (such as the the radial flow rate) into a form that can be used to improve the realism of semi-analytical models.

We conclude from these studies that the expected signature of cosmological inflows in galaxies' metallicity gradients remains unclear theoretically. There is room both for an improved understanding of observed metallicity gradients (Section 2.2), and of what they are telling us about inflows and gas processing.

\section{Extensions to the Equilibrium Model}

Observations indicate that there is more to galaxy metallicities than equilibrium. For example, at a fixed stellar mass, galaxies show finite scatter in gas-phase metallicity (Tremonti et al., 2004; Zahid et al., 2012; Guo et al., 2016), and in star formation rate (Whitaker et al., 2012). Do these observations reflect intrinsic scatter in $\eta$ or $Z_{0}$, or do they indicate departures from equilibrium?

The observed level of scatter is intuitively consistent with the idea that star formation is at least slightly irregular or "bursty" at any mass, which could be associated with departures from equilibrium. For example, if star formation rate is driven by stochastic processes (Gerola et al., 1980) or if the ISM responds slowly to inflow fluctuations, then the galaxy would not be perfectly in equilibrium (Forbes et al., 2014b); this is in fact expected for dwarf galaxies (Lilly et al., 2013; Hopkins et al., 2014: Wise et al., 2014). Alternatively, the burstiness could owe to irregular inflows, which are not themselves completely smooth. Indeed, it has been shown that, by modeling the bursty inflow rates realistically but retaining the assumption that galaxies are in perfect equilibrium with those bursty inflows (which is theoretically supported Rathaus \& Sternberg 2016), the observed level of scatter can be accounted for (Mitra et al., 2016; Dutton et al., 2010). This means that the observed scatter need not reflect truly out-of-equilibrium behavior.

The most important handle on the nature of the observed scatter is the identification of correlated departures from equilibrium, in particular departures from meantrend behavior in $Z$, SFR, and gas fraction at constant $M_{*}$, as quantified by mean scaling relations. These have prompted a range of generalizations to the Equilibrium Model. Before delving into the models, however, we review the observations.

\subsection{The $M_{*}$-Z-SFR Relation: Observations and Intuition}

Ellison et al. (2008) first pointed out that the SDSS MZR has a third-parameter dependence on SFR in the sense that, at a fixed $M_{*}$, galaxies with higher SFR have lower $Z$. Subsequently, other authors have found qualitatively similar results at a 
variety of redshifts (Mannucci et al., 2010; Lara-López et al., 2010; Cresci et al., 2012; Hunt et al., 2012; Henry et al., 2013; Stott et al., 2013; Cullen et al., 2014; Maier et al., 2014; Nakajima \& Ouchi, 2014; de los Reyes et al., 2015; Salim et al., 2015; Kacprzak et al., 2016). There have been suggestions that the SFR-dependence reflects observational systematics or disappears at high redshift (Sánchez et al., 2013; Steidel et al., 2014; Sanders et al., 2015). However, detailed analyses of possible biases have shown that it persists even if a variety of effects is taken into account both in the SDSS sample Andrews \& Martini, 2013; Salim et al., 2014; Telford et al., 2016) and at high redshift (Salim et al., 2015). Moreover, early suggestions that the sign of the effect has a mass dependence in the sense that massive galaxies with enhanced SFR have enhanced metallicity (Yates et al., 2012) may simply reflect tricky selection biases (Salim et al., 2014, 2015). Initial results suggesting that the $M_{*}-Z$-SFR relation is redshift-independent gave rise to the idea that the $M_{*}-Z-S F R$ relation is "fundamental" (Mannucci et al., 2010), which would be powerful if true. However, more recent results indicate that, while the sense of the SFR dependence is unchanged throughout $z=0-2.3$, its strength evolves (Brown et al., 2016; Grasshorn Gebhardt et al., 2016), particularly at high stellar masses $\left(10^{11} M_{\odot}\right.$; Salim et al. 2015).

While the dependence of metallicity on SFR was identified first, it has recently been argued that the dependence on gas fraction is just as strong, if not stronger (Hughes et al., 2013; Bothwell et al., 2013; Lara-López et al., 2013; Bothwell et al., 2016). This result has also been found in cosmological simulations (Lagos et al., 2016). These findings indicate that SFR perturbations really just serve as a proxy for gas supply perturbations. In other words, the $M_{*}-Z$-SFR relation is driven by bursty inflows rather than bursty star formation (Dutton et al., 2010; Mitra et al., 2016). SFR is much easier to measure for large samples, however, and will probably continue to be used as the de facto third parameter for some time.

It thus appears that the $M_{*}-Z$-SFR relation is real, if perhaps a bit elusive. Theoretical explanations generally invoke a picture along the following lines: suppose that a galaxy is growing in a quiescent way that obeys Equations 23. If, for some reason, the gas accretion rate increases, two things will happen. First, the gas mass will grow, likely suppressing the galaxy's metallicity. Second, the SFR will increase owing to the boosted gas density. Finally, the perturbations to $Z$ and SFR subside once the extra gas is processed and the metallicity is diluted by further inflows (Dalcanton, 2007).

Of course, one could also imagine the opposite effect: Dips in the gas accretion rate could lead to suppressed SFR and enhanced metallicity (because the metals generated by ongoing star formation would be less diluted by inflows). The problem with this is that, whereas accretion spikes can increase the size of the gas reservoir and boost SFR on a disk dynamical time ( $\sim 100 \mathrm{Myr})$, accretion dips can only suppress the SFR on the timescale given by the gas mass divided by the SFR. This is, to within a factor of a few, the same as the halo specific growth rate, or 1-10 Gyr (see, for example, Figure 3 of Lilly et al. 2013). It is perhaps for this reason that, observationally, the $M_{*}-Z$-SFR dependence is driven by systems with enhanced 
SFR (Salim et al., 2014; Telford et al., 2016), and evidence for short-term accretion dips is hard to come by.

A variety of theoretical approaches have been used to study the scatter in the $M_{*}$ $Z$-SFR relation and its possible use as a probe of gas inflows. We now summarize key insights gleaned from these efforts.

\subsection{The $M_{*}-Z-S F R$ Relation: Equilibrium Treatments}

\subsubsection{The Power of Assuming $\dot{Z}=\dot{M}_{g}=0$ : Davé et al. 2012}

Inspired by cosmological hydrodynamic simulations, Davé et al. (2012) devised an equilibrium model that relates galaxy growth to host halo growth under the condition that galaxies obey Equations 23. In order to connect halo growth with galaxy growth, they parameterized the impact of photoionization heating, quenching by accretion shocks, and re-accretion of previously ejected gas. They also studied the condition for galaxies to be in gas processing equilibrium, finding that there is a characteristic redshift above which galaxies cannot process their gas as rapidly as they accrete it. The precise redshift depends both on halo mass and on the outflow model because stronger outflows lead galaxies to reach equilibrium sooner. For momentum-driven outflow scalings, $10^{12} M_{\odot}$ halos achieve equilibrium at $z \sim 5$ while galaxies in lower-mass halos $\left(\leq 10^{11} M_{\odot}\right)$ are always in equilibrium (because their outflows are strong). Hence star-forming galaxies are in or near equilibrium for most of cosmic time.

Equations 2, 3 are too simple to permit a third-parameter dependence of $Z$ on SFR, but Davé et al. (2012) did note that such a dependence arises naturally in hydrodynamic simulations and can readily be attributed to weak violations of the condition $\dot{M}_{g}=0$.

The Davé et al. (2012) picture has since been generalized into an eight-parameter model and tuned to match the observed relationships between $M_{*}, Z, \mathrm{SFR}$, and halo mass as well as their redshift dependence and scatter (Mitra et al., 2015, 2016). The resulting fits are excellent, supporting the model's realism. Notably, three of the eight parameters describe the rate at which ejected gas re-accretes and two describe "quenching", or the suppression of inflows into galaxies hosted by massive halos. Such quenching could be associated, for example, with active galactic nuclei or virial shocks. These studies show forcefully that, within a suitable framework, it is possible to use observations of $Z$ and SFR to constrain the efficiency of inflows.

\subsubsection{Relaxing Gas Processing Equilibrium: Lilly et al. 2013}

Initial efforts to interpret ensemble measures of galaxy evolution within an equilibrium framework assumed that the galaxy's gas reservoir does not grow (Finlator et al. 2008; Davé et al., 2011, 2012). However, it was soon realized that relaxing this as- 
sumption and allowing the gas fraction to fluctuate naturally gives rise to a $M_{*-}$ Z-SFR relation that qualitatively resembles observations (Lilly et al., 2013). Rederiving the condition for enrichment equilibrium while relaxing the assumption of gas processing equilibrium leads to a generalization of Equation 3 .

$$
Z_{\mathrm{eq}} \equiv Z_{0}+\frac{y}{(1+\eta)+\varepsilon^{-1}\left[\frac{\mathrm{SFR}}{M_{*}}+(1-R)^{-1} \frac{d \ln \mu}{d t}\right]}
$$

Here, $\varepsilon^{-1}$ is the star formation timescale and $\mu \equiv M_{g} / M_{*}$ is the ratio of gas mass to stellar mass. Equation 4 beautifully connects $Z$, SFR, and $M_{*}$. If the timescale $\varepsilon^{-1}$ for a galaxy to run through its gas reservoir is very short, then $Z$ should not correlate with SFR because the ISM recovers quickly from perturbations (by processing gas efficiently). In this case, the gas mass is constant and Equation 4 reduces to Equation 3 On the other hand, if star formation is less efficient ( $\varepsilon^{-1}$ is longer), then the ISM can be out of gas processing equilibrium for some time. In this case, a high specific star formation rate $\mathrm{SFR} / M_{*}$ is associated with a low metallicity as observed, irrespective of the relationship between the inflow and gas processing rates.

After presenting this model, Lilly et al. (2013) used it to draw a number of inferences from observations. In particular:

1. If the model is tuned to match the observed MZR at $z=0$, then it immediately predicts the strong observed dependence of stellar mass on halo mass (Moster et al. 2010), suggesting that the latter trend may be governed entirely by the details of feedback and gas processing that occur deep within the ISM. This does not rule out the possible importance of suppression or feedback processes that occur in the CGM, but it indicates that they are not required.

2. The model can readily be tuned to match observations of the $M_{*}$-Z-SFR dependence, and it predicts that this relation does not evolve with redshift unless the parameters governing gas processing (i.e., $\eta, Z_{0}$, and $\varepsilon$ ) also evolve. By contrast, it predicts that the MZR's normalization increases with time because high-redshift galaxies have systematically higher specific star formation rates.

The gas regulator model contains a number of approximations, chief among them the assumption of enrichment equilibrium. It is naturally of interest to ask whether the model describes what happens within a less approximate framework. Pipino et al. (2014) tested the Lilly et al. (2013) model using exact analytical expressions for the evolution of gas-phase metallicity, gas mass, and star formation rate, finding excellent agreement across a wide range of potential growth histories. This suggests that the gas regulator model is a powerful generalization of the equilibrium model introduced by Davé et al. (2012). Not surprisingly, it is now being tested and applied in a wide variety of contexts (for example, Birrer et al. 2014; Dekel \& Mandelker 2014; Bouché \& Blandin 2015; Wu et al. 2016).

To date, there has been no effort to determine whether the Lilly et al. (2013) model describes what happens within three-dimensional hydrodynamic simulations or semi-analytical models; this may be a promising avenue for future research. 


\subsection{The $M_{*}-Z-S F R$ Relation: Non-equilibrium Treatments}

\subsubsection{An Analytical Model Without Equilibrium: Dayal et al. 2013}

Can the observed $M_{*}$-Z-SFR relation can be explained without any explicit assumption of equilibrium? In a purely analytical study, Dayal et al. (2013) presented a minimal model for galaxy growth in which the evolution of the stellar, gas, and metal reservoirs depends only on the unknown gas accretion and outflow rates. They assume that outflow and infall rates depend only on stellar mass, that the metal yield $y$ is constant, and that inflows are pristine. They then show that they can fit the model's governing parameters using the the observed $M_{*}$-Z-SFR relation (Mannucci et al., 2010) to achieve good agreement.

In common with Lilly et al. (2013), this model predicts no evolution in the overall $M_{*}$-Z-SFR relation for a given set of parameters, but it has the advantage that it assumes neither gas processing or enrichment equilibrium. Nonetheless, a massdependent balance between inflows and outflows emerges as a central prediction. Massive galaxies have weak winds and rapidly achieve enrichment equilibrium (similar to Equation 3, although they do not impose this). By contrast, low-mass galaxies lose most of their metals through much stronger outflows and are hence more susceptible to dilution.

In fact, they show that, subject to their modeling assumptions, the infall rate and its mass dependence can be inferred directly from the observed $M_{*}$-Z-SFR relation. Intriguingly, the inferred outflow rate $\eta$ varies with mass in a way that follows expectations from momentum-driven outflow models (Murray et al.,2005). The model can also accommodate high-redshift observations, with the result that gas fractions and inflow rates were previously higher while outflow efficiencies were essentially the same (Hunt et al., 2016).

Their model also naturally reproduces the observed relation between oxygen abundance and neutral gas fraction (Hughes et al., 2013). This finding reinforces the tight connection between metallicity and gas fraction that emerges in any plausible model of galaxy growth. It would be interesting to ask whether re-writing the Dayal et al. (2013) model to match the observed $M_{*}$-SFR-gas fraction relation automatically recovers the $Z$ dependence. Likewise, it would be interesting to quantify precisely how close the galaxies are to enrichment and gas processing equilibrium in the Dayal et al. (2013) model once the inflow and outflow parameters are tuned to match observations.

\subsubsection{Stochastic Accretion Histories and Scatter: Forbes et al. 2014}

Observationally, there is residual scatter in $Z$ even after the SFR dependence is removed (Salim et al., 2014, 2015). Can this scatter be used as a complementary probe of inflows and gas processing? This question is taken up by Forbes et al. (2014b), who propose that, even if individual galaxies are not in equilibrium, they may be close enough to it that ensemble statistics such as the slope of the Z-SFR relation 
are in equilibrium. They explore this idea by modeling galaxy growth as a connected sequence of intervals whose constant duration is given by the "coherence time" $t_{\text {coherence. }}$. At the start of each interval, a new accretion rate is drawn from a log-normal distribution with fixed median and scatter. The galaxy then uses star formation and outflows to respond to the new accretion rate on a "loss timescale" $t_{\text {loss }}$, which incorporates gas losses to both outflows and star formation. If $t_{\text {loss }}$ is short compared to other timescales, then galaxies are always in equilibrium. Conversely, if $t_{\text {loss }}$ is long, then they retain a "memory" of previous accretion episodes.

The model's parameters can be tuned to yield qualitative agreement with the observed $M_{*}$-Z-SFR relations. This has two important implications:

1. Galaxy metallicities, when combined with measures of $M_{*}$ and SFR, may probe both the mean and the distribution in gas accretion rates;

2. As in Daval et al. (2013), observations do not require galaxies to obey either of the equilibrium relations (Equations 2-33) explicitly, although in practice they may be nearly in equilibrium.

The price for relaxing the assumption of equilibrium is that this model is more complicated than the Lilly et al. (2013) or Davé et al. (2012) models. It would therefore be useful to ask how close galaxies are to equilibrium when this model is tuned to reproduce observations. Moreover, as the authors point out, the Forbes et al. (2014b) model for inflow fluctuations is extremely simple, invoking only a single coherence timescale. In reality, inflows fluctuate on a range of timescales. It would therefore be useful to generalize this aspect of the model for improved realism.

\subsubsection{Cosmological Hydrodynamic Simulations}

Cosmological hydrodynamic simulations and semi-analytical models make fewer assumptions regarding inflows and gas processing than analytical models (Somerville \& Davé, 2015), and are hence a particularly realistic framework for studying the $M_{*}-Z$-SFR relation. Reassuringly, they inevitably predict relations that qualitatively resemble observations (Davé et al., 2011; Yates et al., 2012; Obreia et al., 2014; de Rossi et al. 2015; Kacprzak et al., 2016; Lagos et al., 2016; Cousin et al., 2016).

In order to understand this prediction better, Lagos et al. (2016) apply a principal component analysis to the predicted ensemble distribution of global galaxy attributes in the EAGLE simulations (Schaye et al., 2015). In agreement with previous work, they identify a particularly thin plane in the space of $M_{*}$, SFR, and neutral+molecular gas fraction where simulated galaxies tend to live. Moreover, they show that this plane is observed. They interpret its existence as an indication that galaxies live in a slowly-evolving balance between gas accretion and gas processing reminiscent of Equation 2 Interestingly, the predicted scatter about this plane tightens if stellar feedback assumed in the model is stronger. This could readily reflect the fact that fresh inflows drive galaxies back to their equilibrium state more quickly if the gas fraction is lower. 
Just as the statistics of the EAGLE galaxies imply a balance between gas inflows and processing, they also imply a balance between dilution and enrichment: the predicted metallicity is correlated with the gas fraction in the observed sense, namely that galaxies with high gas fractions have systematically low metallicities. Interestingly, however, it is found that $Z$ contributes less to the overall variation in galaxy properties than $M_{*}$, SFR, and gas fraction: whereas the latter three attributes dominate the first principal component, $Z$ only enters into the second. This qualitatively supports observational suggestions that gas fraction is more fundamental than metallicity (Hughes et al., 2013; Bothwell et al,, 2013; Lara-López et al., 2013; Bothwell et al.,2016). Further theoretical work is needed in order to understand why gas fraction is more fundamental.

Unlike recent high-resolution simulations of individual galaxies (Christensen et al. 2016; Ma et al., 2016), the study presented in Lagos et al. (2016) stops short of directly measuring the flow of gas and metals through the simulated galaxies because it is based on a principal-component analysis, which leverages the statistical advantage that lower-resolution cosmological simulations have over high-resolution "zoom" studies. This means that they do not ask whether galaxies keep gas mass or gas fraction constant, or how $Z_{0}$ varies with mass and redshift. It would therefore be interesting to merge the two approaches by quantifying the expected flow of gas and metals in cosmological simulations in order to understand in more detail how they give rise to the $M_{*}-Z$-SFR-gas fraction relation.

\subsubsection{An Analytical Model Without Inflows: Magrini et al. 2012}

Thus far, all of the models for the $M_{*}$-Z-SFR relation that we have discussed invoke inflows, giving the impression that cosmological inflows of pristine gas are an unavoidable inference. A different perspective is offered by Magrini et al. (2012), who construct analytic, one-zone, closed-box evolutionary models that qualitatively match the observed $M_{*}-Z$-SFR relation. The key ingredient in their model is a distinction between "active" and "passive" modes of star formation: active-mode galaxies have high-density molecular clouds whose collisions trigger vigorous star formation while the clouds in passive-mode galaxies are more diffuse. When they confront their model with observations, they find that most high-redshift galaxies and the occasional, unusually active low-redshift ones can be identified with the active mode while quiescent systems can be identified with the passive mode.

While it is interesting that the model in Magrini et al. (2012) contains observations within its accessible parameter space, the discussion leaves several basic questions unaddressed. First, the model does not predict the distribution of active and passive-mode systems or its evolution. Hydrodynamic simulations attribute the prevalence of active-mode star-formers at high redshift and the scaling relations that they obey to high gas accretion rates and the detailed way in which galaxies achieve an equilibrium with those inflows (Forbes et al., 2014a; Lagos et al., 2016). By contrast (and similarly to Daval et al.2013), Magrini et al. (2012) attribute the observed scaling relationships to unexplained variations in initial conditions, evolutionary 
state, and ISM properties. Second, the lack of inflows means that the Magrini et al. (2012) predicts stellar metallicity distributions that conflict with local observations (Section 1). Future work that includes inflows following cosmological expectations may reconcile this model with simulations.

\section{Summary}

Over the past 20 years, a marked dichotomy has emerged that distinguishes between how dark matter halos and galaxies grow. While halos do much or most of their growing by merging with other halos, star-forming galaxies with stellar masses below $\sim 10^{11} M_{\odot}$ acquire most of their baryons through relatively smooth gas flows. Theoretical models predict that these inflows leave a number of observable signatures in galaxies' gas-phase metallicities. Mergers leave a statistically-detectable imprint both on galaxies' central gas-phase metallicities and on their radial metallicity gradients, but they are not the primary mechanism for delivering fresh gas to galaxies.

Radial metallicity gradients are a promising probe of inflows because they are expected to flatten once previously-ejected gas begins to re-accrete. This is because metals that form and are ejected in a galaxy's core are "spun up" by the halo and re-distributed to large radii. To date, however, radial metallicity gradients in nearby galaxies have not been demonstrated to require inflows. Instead, they have been attributed either to radial gradients in ISM properties such as the strength of outflows, or to a radially-varying "evolutionary state" in which annuli at large radii are simply less evolved. These conflicting interpretations point to the need for further theoretical inquiry into how low-redshift observations ought to be interpreted.

At high redshifts, simulations predict that metallicity gradients may have been stronger than in the local Universe, possibly because of the weaker role of recycled gas and mergers at early times. Meanwhile, observations seem split between lensed galaxies with generally strong gradients and unlensed galaxies with weak ones. Further observational work is evidently required in order to clarify the level of agreement with theoretical models.

Moving from radial gradients to ensemble statistics, the interplay between inflows and gas processing leads to a rich phenomenology in which $M_{*}, Z$, SFR, and gas fraction are tightly coupled. The resulting correlations are observed out to at least $z=2$, and they are being disentangled via a wide variety of theoretical studies.

Analytical treatments have shown that the observed $M_{*}$-Z-SFR correlation follows naturally from the assumption that gas processing is nearly in equilibrium with inflows, and that enrichment is nearly in equilibrium with dilution. Models that do not explicitly assume equilibrium tend to recover it once their parameters are tuned to match observations. Consequently, they can be used to infer inflow rates (in addition to other physical parameters) from the $M_{*}-Z$-SFR relation. They have further shown that there is a tight connection between $Z$, SFR, and gas fraction in the sense that tuning the model to match one observable often yields agreement with another, 
free of charge. Detailed measurement of inflows and outflows in high-resolution numerical simulations support the importance of quasi-equilibrium behavior governed by outflows whose efficiency decreases with mass. Meanwhile, cosmological simulations (and at least one semi-analytical model) find that a realistic $M_{*}$-Z-SFRgas fraction relation arises naturally within sufficiently realistic frameworks. This ensemble statistical behavior has been interpreted as support for predominantly quiescent, equilibrium-like galaxy growth.

The progress that has been made in understanding the $M_{*}$-Z-SFR relation, while promising, does not yet explain everything. Even after removing the dependence of $Z$ on specific star formation rate $\mathrm{SFR} / M_{*}$, the residual observed scatter in $Z$ at a given $M_{*}$ remains substantial (Salim et al., 2014, 2015). If the SFR (or gas fraction) dependence can largely be accounted for by relaxing the assumption of gas processing equilibrium, then does the residual scatter indicate departure from enrichment equilibrium or intrinsic scatter in $\eta$ or $Z_{0}$ ? This will be a useful question to take up in future theoretical studies.

In summary, it is now widely-recognized that star-forming galaxies do most of their growing in a quiescent mode where the global SFR, $Z$, and gas fraction constantly adjust on a relatively short timescale to reflect the influence of inflows. Further study of the flow of gas and metals in numerical simulations is required in order to improve our understanding of equilibrium growth, but its broad role is now beyond dispute. From the beginning, measurements of metallicities have driven the development of this paradigm. There is no doubt that they will continue to do so.

Acknowledgements The author thanks R. Davé and A. Fox for offering him the opportunity to contribute this chapter, and for their patience as he drafted it. Additional thanks go to A. Klypin for detailed and honest comments on an early version.

\section{References}

Andrews, B. H., \& Martini, P. 2013, ApJ, 765, 140

Behroozi, P. S., Wechsler, R. H., \& Conroy, C. 2013, ApJ, 770, 57

Birrer, S., Lilly, S., Amara, A., Paranjape, A., \& Refregier, A. 2014, ApJ, 793, 12

Bothwell, M. S., Maiolino, R., Kennicutt, R., et al. 2013, MNRAS, 433, 1425

Bothwell, M. S., Maiolino, R., Cicone, C., Peng, Y., \& Wagg, J. 2016, arXiv:1606.04102

Bouché, N., Dekel, A., Genzel, R., et al. 2010, ApJ, 718, 1001

Bouché, N., \& Blandin, S. 2015, Revolution in Astronomy with ALMA: The Third Year, 499, 63

Brook, C. B., Stinson, G., Gibson, B. K., et al. 2012, MNRAS, 419, 771

Brook, C. B., Stinson, G., Gibson, B. K., et al. 2014, MNRAS, 443, 3809

Brown, J. S., Martini, P., \& Andrews, B. H. 2016, MNRAS, 458, 1529

Carton, D., Brinchmann, J., Wang, J., et al. 2015, MNRAS, 451, 210

Casuso, E., \& Beckman, J. E. 2004, A\&A, 419, 181 
Christensen, C. R., Davé, R., Governato, F., et al. 2016, ApJ, 824, 57

Cooper, M. C., Tremonti, C. A., Newman, J. A., \& Zabludoff, A. I. 2008, MNRAS, 390,245

Cousin, M., Buat, V., Boissier, S., et al. 2016, A\&A, 589, A109

Cresci, G., Mannucci, F., Maiolino, R., et al. 2010, Nature, 467, 811

Cresci, G., Mannucci, F., Sommariva, V., et al. 2012, MNRAS, 421, 262

Cullen, F., Cirasuolo, M., McLure, R. J., Dunlop, J.S., \& Bowler, R.A.A. 2014, MNRAS, 440, 2300

Dalcanton, J. J. 2007, ApJ, 658, 941

Davé, R. 2008, MNRAS, 385, 147

Davé, R., Finlator, K., \& Oppenheimer, B. D. 2011, MNRAS, 416, 1354

Davé, R., Finlator, K., \& Oppenheimer, B. D. 2012, MNRAS, 421, 98

Davé, R., Thompson, R. J., \& Hopkins, P. F. 2016, arXiv:1604.01418

Dayal, P., Ferrara, A., \& Dunlop, J. S. 2013, MNRAS, 430, 2891

Dekel, A., \& Birnboim, Y. 2006, MNRAS, 368, 2

Dekel, A., \& Mandelker, N. 2014, MNRAS, 444, 2071

de los Reyes, M. A., Ly, C., Lee, J. C., et al. 2015, AJ, 149, 79

De Rossi, M. E., Theuns, T., Font, A. S., \& McCarthy, I. G. 2015, MNRAS, 452, 486

Dutton, A. A., van den Bosch, F. C., \& Dekel, A. 2010, MNRAS, 405, 1690

Ellison, S. L., Patton, D. R., Simard, L., \& McConnachie, A. W. 2008, ApJL, 672, L107

Erb, D. K., Shapley, A. E., Pettini, M., et al. 2006, ApJ, 644, 813

Feldmann, R. 2013, MNRAS, 433, 1910

Faisst, A. L., Capak, P. L., Davidzon, I., et al. 2016, ApJ, 822, 29

Finlator, K., \& Davé, R. 2008, MNRAS, 385, 2181

Forbes, J. C., Krumholz, M. R., Burkert, A., \& Dekel, A. 2014a, MNRAS, 438, 1552

Forbes, J. C., Krumholz, M. R., Burkert, A., \& Dekel, A. 2014b, MNRAS, 443, 168

Fu, J., Kauffmann, G., Huang, M.-1., et al. 2013, MNRAS, 434, 1531

Genel, S. 2016, ApJ, 822, 107

Gerola, H., Seiden, P. E., \& Schulman, L. S. 1980, ApJ, 242, 517

Grasshorn Gebhardt, H. S., Zeimann, G. R., Ciardullo, R., et al. 2016, ApJ, 817, 10

Grønnow, A. E., Finlator, K., \& Christensen, L. 2015, MNRAS, 451, 4005

Gnedin, N. Y. 2000, ApJ, 542, 535

Guo, Y., Koo, D. C., Lu, Y., et al. 2016, ApJ, 822, 103

Henriques, B. M. B., White, S. D. M., Thomas, P. A., et al. 2013, MNRAS, 431, 3373

Henry, A., Martin, C. L., Finlator, K., \& Dressler, A. 2013, ApJ, 769, 148

Ho, I.-T., Kudritzki, R.-P., Kewley, L. J., et al. 2015, MNRAS, 448, 2030

Hopkins, P. F., Kereš, D., Oñorbe, J., et al. 2014, MNRAS, 445, 581

Hughes, T. M., Cortese, L., Boselli, A., Gavazzi, G., \& Davies, J. I. 2013, A\&A, 550, A115

Hunt, L., Magrini, L., Galli, D., et al. 2012, MNRAS, 427, 906

Hunt, L., Dayal, P., Magrini, L., \& Ferrara, A. 2016, MNRAS, 
Jones, T., Ellis, R. S., Richard, J., \& Jullo, E. 2013, ApJ, 765, 48

Kacprzak, G. G., van de Voort, F., Glazebrook, K., et al. 2016, ApJL, 826, L11

Kereš, D., Katz, N., Weinberg, D. H., \& Davé, R. 2005, MNRAS, 363, 2

Kewley, L. J., Rupke, D., Zahid, H. J., Geller, M. J., \& Barton, E. J. 2010, ApJL, 721, L48

Kirby, E. N., Cohen, J. G., Smith, G. H., et al. 2011, ApJ, 727, 79

Köppen, J., \& Edmunds, M. G. 1999, MNRAS, 306, 317

Lagos, C. d. P., Theuns, T., Schaye, J., et al. 2016, MNRAS, 459, 2632

Lara-López, M. A., Cepa, J., Bongiovanni, A., et al. 2010, A\&A, 521, L53

Lara-López, M. A., Hopkins, A. M., López-Sánchez, A. R., et al. 2013, MNRAS, 433, L35

Lilly, S. J., Carollo, C. M., Pipino, A., Renzini, A., \& Peng, Y. 2013, ApJ, 772, 119

Ma, X., Hopkins, P. F., Faucher-Giguère, C.-A., et al. 2016, MNRAS, 456, 2140

Magrini, L., Hunt, L., Galli, D., et al. 2012, MNRAS, 427, 1075

Maier, C., Lilly, S. J., Ziegler, B. L., et al. 2014, ApJ, 792, 3

Maiolino, R., Nagao, T., Grazian, A., et al. 2008, A\&A, 488, 463

Mannucci, F., Cresci, G., Maiolino, R., Marconi, A., \& Gnerucci, A. 2010, MNRAS, 408, 2115

Martin, C. L., Kobulnicky, H. A., \& Heckman, T. M. 2002, ApJ, 574, 663

Michel-Dansac, L., Lambas, D. G., Alonso, M. S., \& Tissera, P. 2008, MNRAS, 386, L82

Mitra, S., Davé, R., \& Finlator, K. 2015, MNRAS, 452, 1184

Mitra, S., Davé, R., Simha, V., \& Finlator, K. 2016, arXiv:1606.07436

Moster, B. P., Somerville, R. S., Maulbetsch, C., et al. 2010, ApJ, 710, 903

Mouhcine, M., Baldry, I. K., \& Bamford, S. P. 2007, MNRAS, 382, 801

Muratov, A. L., Kereš, D., Faucher-Giguère, C.-A., et al. 2015, MNRAS, 454, 2691

Murray, N., Quataert, E., \& Thompson, T. A. 2005, ApJ, 618, 569

Nakajima, K., \& Ouchi, M. 2014, MNRAS, 442, 900

Nelson, D., Vogelsberger, M., Genel, S., et al. 2013, MNRAS, 429, 3353

Nelson, D., Genel, S., Vogelsberger, M., et al. 2015, MNRAS, 448, 59

Obreja, A., Brook, C. B., Stinson, G., et al. 2014, MNRAS, 442, 1794

Okamoto, T., Gao, L., \& Theuns, T. 2008, MNRAS, 390, 920

Oppenheimer, B. D., Davé, R., Kereš, D., et al. 2010, MNRAS, 406, 2325

Pagel, B. E. J., \& Patchett, B. E. 1975, MNRAS, 172, 13

Papovich, C., Finkelstein, S. L., Ferguson, H. C., Lotz, J. M., \& Giavalisco, M. 2011, MNRAS, 412, 1123

Peng, Y.-j., \& Maiolino, R. 2014, MNRAS, 438, 262

Pilkington, K., Few, C. G., Gibson, B. K., et al. 2012, A\&A, 540, A56

Pipino, A., Lilly, S. J., \& Carollo, C. M. 2014, MNRAS, 441, 1444

Rathaus, B., \& Sternberg, A. 2016, MNRAS, 458, 3168

Rupke, D. S. N., Kewley, L. J., \& Chien, L.-H. 2010b, ApJ, 723, 1255

Rupke, D. S. N., Kewley, L. J., \& Barnes, J. E. 2010a, ApJL, 710, L156

Salim, S., Lee, J. C., Ly, C., et al. 2014, ApJ, 797, 126

Salim, S., Lee, J. C., Davé, R., \& Dickinson, M. 2015, ApJ, 808, 25

Sanders, R. L., Shapley, A. E., Kriek, M., et al. 2015, ApJ, 799, 138 
Schaye, J., Crain, R. A., Bower, R. G., et al. 2015, MNRAS, 446, 521

Schmidt, M. 1963, ApJ, 137, 758

Schlesinger, K. J., Johnson, J. A., Rockosi, C. M., et al. 2012, ApJ, 761, 160

Sánchez, S. F., Rosales-Ortega, F. F., Jungwiert, B., et al. 2013, A\&A, 554, A58

Scudder, J. M., Ellison, S. L., Torrey, P., Patton, D. R., \& Mendel, J. T. 2012, MNRAS, 426, 549

Scudder, J. M., Patton, D. R., Ellison, S. L., Torrey, P., \& Mendel, J. T. 2013, Galaxy

Mergers in an Evolving Universe, ASP Conf. Ser. 477, San Francisco:ASP, p241

Somerville, R. S., \& Davé, R. 2015, ARA\&A, 53, 51

Springel, V., White, S. D. M., Jenkins, A., et al. 2005, Nature, 435, 629

Steidel, C. C., Rudie, G. C., Strom, A. L., et al. 2014, ApJ, 795, 165

Stott, J. P., Sobral, D., Bower, R., et al. 2013, MNRAS, 436, 1130

Tacconi, L. J., Neri, R., Genzel, R., et al. 2013, ApJ, 768, 74

Telford, O. G., Dalcanton, J. J., Skillman, E. D., \& Conroy, C. 2016, ApJ, 827, 35

Tinsley, B. M., \& Larson, R. B. 1978, ApJ, 221, 554

Tinsley, B. M. 1980, Fundamentals of Cosmic Physics, 5, 287

Torrey, P., Cox, T. J., Kewley, L., \& Hernquist, L. 2012, ApJ, 746, 108

Tremonti, C. A., Heckman, T. M., Kauffmann, G., et al. 2004, ApJ, 613, 898

van Dokkum, P. G. 2008, ApJ, 674, 29-50

Veilleux, S., Cecil, G., \& Bland-Hawthorn, J. 2005, ARA\&A, 43, 769

Weinmann, S. M., Pasquali, A., Oppenheimer, B. D., et al. 2012, MNRAS, 426, 2797

Whitaker, K. E., van Dokkum, P. G., Brammer, G., \& Franx, M. 2012, ApJL, 754, L29

White, S. D. M., \& Frenk, C. S. 1991, ApJ, 379, 52

White, S. D. M., \& Rees, M. J. 1978, MNRAS, 183, 341

White, C. E., Somerville, R. S., \& Ferguson, H. C. 2015, ApJ, 799, 201

Wise, J. H., Demchenko, V. G., Halicek, M. T., et al. 2014, MNRAS, 442, 2560

Wolfe, A. M., Gawiser, E., \& Prochaska, J. X. 2005, ARA\&A, 43, 861

Wu, H.-Y., Doré, O., \& Teyssier, R. 2016, arXiv:1607.02546

Wuyts, E., Wisnioski, E., Fossati, M., et al. 2016, ApJ, 827, 74

Yates, R. M., Kauffmann, G., \& Guo, Q. 2012, MNRAS, 422, 215

Yuan, T.-T., Kewley, L. J., Swinbank, A. M., Richard, J., \& Livermore, R. C. 2011, ApJL, 732, L14

Zahid, H. J., Bresolin, F., Kewley, L. J., Coil, A. L., \& Davé, R. 2012, ApJ, 750, 120

Zaritsky, D., Kennicutt, R. C., Jr., \& Huchra, J. P. 1994, ApJ, 420, 87 